\title{
RESEARCH
}

Open Access

\section{Interleukin-9 promotes intestinal barrier injury of sepsis: a translational research}

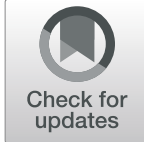

Jia-Kui Sun ${ }^{1,2 \dagger}$, Jing Zhou ${ }^{1 \dagger}$, Xin-Pei Sun ${ }^{3}$, Xiao Shen ${ }^{2}$, Dong-Mei Zhu' ${ }^{1}$, Xiang Wang ${ }^{2^{*}}$, Su-Ming Zhou ${ }^{1^{*}}$ and

Xin-Wei $\mathrm{Mu}^{2^{*}}$ (D)

\begin{abstract}
Background: Sepsis is a life-threatening organ dysfunction caused by a dysregulated host response to infection. Intestinal mucosal barrier injury is one of the important manifestations of sepsis. Interleukin-9 (IL-9) and IL-9producing CD4(+) T cells were emerging pro-inflammatory mediators with development of intestinal injury. However, it is unclear whether IL-9 is related to the intestinal barrier injury of sepsis.
\end{abstract}

Methods: To investigate the roles of IL-9-producing CD4(+) T cells and IL-9 in the process of barrier injury in sepsis, serum IL-9-producing CD4(+) T cell percentages, IL-9, and D-lactate levels were measured in septic patients and controls. The markers of barrier function in serum and intestinal tissue were also collected in septic rats. Moreover, the barrier injury degree and survival rate of septic rats were also investigated after increasing or interfering with IL9 expression.

Results: The serum IL-9-producing CD4(+) T cell percentages, IL-9, and D-lactate levels were significantly higher in septic patients or rats than those in controls. IL-9-producing CD4(+) T cells and IL-9 levels were positively correlated with D-lactate levels and had a high predictive value of 28-day mortality in septic patients. The non-survivors had significantly higher serum T cell percentages, IL-9, and D-lactate levels compared with survivors. In septic rats, IL-9 increased the expression levels of D-lactate, whereas that decreased the expression levels of zonula occludens 1. Moreover, the barrier injury was aggravated or alleviated by increasing or interfering with IL-9 expression, respectively. Survival rate analysis also showed that IL-9 decreased the 14-day survival rate of septic rats.

Conclusion: IL-9 is closely related to intestinal mucosal barrier injury and mortality in sepsis. IL-9 blockade has the potential to improve the barrier injury in sepsis.

Trial registration: The study was registered at ClinicalTrials.gov (ID: NCT03791866, Date: December 2018).

Keywords: IL-9-producing CD4(+) T cells, IL-9, Imucosal barrier, Sepsis

\footnotetext{
* Correspondence: njdrwx2016@163.com; zhousmco@aliyun.com; njdrmxw2012@163.com

${ }^{\dagger}$ Jia-Kui Sun and Jing Zhou contributed equally to this work.

2Department of Critical Care Medicine, Nanjing First Hospital, Nanjing

Medical University, 68 Changle Road, Nanjing 210006, Jiangsu Province,

China

'Department of Geriatrics Intensive Care Unit, The First Affiliated Hospital of

Nanjing Medical University (Jiangsu Province People's Hospital), 300

Guangzhou Road, Nanjing 210029, Jiangsu Province, China

Full list of author information is available at the end of the article
}

(C) The Author(s). 2021 Open Access This article is licensed under a Creative Commons Attribution 4.0 International License, which permits use, sharing, adaptation, distribution and reproduction in any medium or format, as long as you give appropriate credit to the original author(s) and the source, provide a link to the Creative Commons licence, and indicate if changes were made. The images or other third party material in this article are included in the article's Creative Commons licence, unless indicated otherwise in a credit line to the material. If material is not included in the article's Creative Commons licence and your intended use is not permitted by statutory regulation or exceeds the permitted use, you will need to obtain permission directly from the copyright holder. To view a copy of this licence, visit http://creativecommons.org/licenses/by/4.0/ The Creative Commons Public Domain Dedication waiver (http://creativecommons.org/publicdomain/zero/1.0/) applies to the data made available in this article, unless otherwise stated in a credit line to the data. 


\section{Background}

Sepsis is a life-threatening organ dysfunction caused by a dysregulated host response to infection $[1,2]$. Previous studies have indicated that immune imbalance might play an important role in the development of sepsis [3, 4], which is closely associated with the injury of intestinal mucosal barrier in sepsis $[5,6]$. Intestinal tract is a main immune organ supplying an original barrier against pathogenic microorganism [3, 5]. Intestinal barrier injury was often caused by an inflammatory reaction, severe trauma, shock, infection, pancreatitis, and other critical illnesses [3, 5]. Accompanying by sepsis, intestinal epithelial cell damaged, mucosal permeability increased, intestinal flora translocated, and then further intestinal original infection developed [3, 5]. Therefore, intestinal dysfunction is considered as "motor of multiple organ failure." However, the precise mechanisms of intestinal barrier damage caused by sepsis are still unclear.

In addition to the conventional $\mathrm{T}$ helper (Th) 1/ Th2 and Th17/T regulatory (Treg) lymphocytes, a latest described Th subset, IL-9-producing CD4(+) T helper cells (Th9), was reported to induce infection, tumor, asthma, inflammatory bowel disease, and other allergic or autoimmune diseases mainly through the interleukin-9 (IL-9) pathway [7-10]. Th9 cells are mainly derived from naïve $\mathrm{T}$ cells under the combined stimulation of IL- 4 and TGF- $\beta$, which are characterized by the secretion of IL- 9 and IL-10 [7, 10]. Although IL-9 was also produced by other $\mathrm{T}$ helper cells, the Th9 cells were confirmed to be the main cells secreting IL-9 [10]. IL-9 has biological effects on various immune cell types that are involved in the development of inflammation by Janus kinase-signal transduction and transcription activation [11, 12]. Nalleweg et al. [7] found that IL-9 receptor was overexpressed on gut epithelial cells, and the IL-9 level was significantly correlated with intestinal local inflammation in ulcerative colitis. Accordingly, IL-9producing CD4(+) T cells and IL-9 appear to be new therapeutic targets in allergic or autoimmune diseases [12-15].

Sepsis is characterized by acute severe systemic inflammation and infection, which is distinct from the chronic local inflammation in autoimmune diseases. It is unclear whether IL-9-producing CD4(+) T cells and IL-9 are related to the acute injury of the intestinal mucosal barrier in sepsis. The purpose of this study was to investigate the relationships between IL-9-producing CD4(+) T cells/IL-9 and intestinal barrier injury in clinical septic patients and then verify the roles of IL-9 in the intestinal injury in animal experiments. Overall, our results demonstrated that IL-9 is involved in the damage of the intestinal mucosal barrier in sepsis.

\section{Methods}

\section{Human patients and controls}

Thirty-six adult septic patients (age 18-70 years) admitted into the intensive care unit (ICU) of Nanjing First Hospital were included in this study. The diagnostic criteria for sepsis were in accordance with the surviving sepsis guidelines [1]. Patients with ileus or digestive tract hemorrhage, inflammatory bowel disease, severe abdominal hypertension, chronic organ dysfunction (e.g., hepatic or renal dysfunction), malnutrition, or immunodeficiency, and patients with a history of long-term use of hormones were excluded. All patients received standard treatments for sepsis as needed, including antimicrobial therapy, fluid resuscitation, vasopressor administration, oxygen administration, mechanical ventilation, glucose control, and renal replacement therapy.

Fifteen baseline data (including age, sex, body-mass index, disease severity scores) matched adult non-septic critical patients of our ICU were selected as controls. This study protocol was approved by the Institutional Ethics Committee of Nanjing First Hospital (Approval Number: KY20180713-01), and informed consent was obtained from each patient's first-degree relatives according to the Declaration of Helsinki. This study was also registered at ClinicalTrials.gov (ID: NCT03791866). The baseline data, including age, sex, body-mass index, etiology of sepsis, disease categories of controls, Acute Physiology and Chronic Health Evaluation II (APACHE II) scores, and Sequential Organ Failure Assessment (SOFA) scores for both septic and control patients, were collected. The IL-9-producing CD4(+) T cell percentages, IL-9, and D-lactate (biomarker of mucosal barrier function) [5] levels in peripheral blood were tested on days 1,3 , and 7 after admission. In addition, clinical outcome variables including 28-day mortality and ICU duration (days) were recorded.

\section{Sepsis model and groups}

To verify the precise roles of IL-9 in the intestinal injury of sepsis, we performed the pre-clinical experiments using SD rats. Adult SD rats (weight 180-220 g, about 6-8 weeks old) were purchased from the experimental animal center of Zhejiang Academy of Medical Sciences (Zhejiang, China). Sepsis was induced by cecum ligation and puncture (CLP) as reported in previous studies [16, 17]. The rats were anesthetized by intraperitoneal injection of $10 \%$ chloral hydrate at a dose of $0.3 \mathrm{ml} / 100 \mathrm{~g}$. An incision of $2.5 \mathrm{~cm}$ from middle abdomen was performed under sterile conditions. The cecum was ligated in the middle segment with 3-0 silk and then was punctured with a 22-gauge needle. After that, the cecum was placed back into the abdominal cavity, and the abdomen was closed. Sham-operated (control) rats received identical laparatomy, where the cecum was exposed without 
ligature or puncture. Normal saline and antibiotic were administered to prevent hypotension.

Rats were randomly assigned to the four groups $(n=$ 12 in each group). In addition to the sepsis group and control group (sham operation group), we also added the sepsis+sh-IL-9 (IL-9 inhibition) group and sepsis+IL9 (IL-9 overexpression) group in the animal experiments. These groups were necessary to observe the effects of IL-9 on the intestinal injury of sepsis from various perspectives. Rats in the sepsis+sh-IL-9 group received adeno-associated virus (AAV) of IL-9 interference, whereas the sepsis+IL-9 group received AAV of IL-9 overexpression by intraperitoneal injection. The AAV was produced according to a previous procedure [18]. Briefly, IL-9 shRNA cassette or cDNA clone fragment encoding IL-9 were constructed into rAAV plasmid, respectively (Genepharma, Shanghai, China). Then the plasmid was co-transfected with AAV package and shuttle plasmids into AAV-293 cell line. The AAV virus particle was harvested after using ammonium sulfate precipitation and iodixanol continuous gradient centrifugation. Genome titers of the AAV particles were determined by real-time polymerase chain reaction. The AAV particle was stored in $-80^{\circ} \mathrm{C}$ refrigerator. The dosage of AAV particle was $100 \mu \mathrm{l}$, and the titers were $2 \times 10^{11} \mathrm{vg} /$ $\mathrm{ml}$ [18]. Sepsis was induced 7 days after AAV injection.

Rats were sacrificed on the 7th day after sepsis induction. The rats were anesthetized by intraperitoneal injection of $10 \%$ chloral hydrate at a dose of $0.3 \mathrm{ml} / 100 \mathrm{~g}$ before taking serum and intestinal tissue. Blood was collected in tubes with heparin after cardiac puncture, centrifuged $(10 \mathrm{~min}, 1500 \times \mathrm{g}$ ). Colons were collected from the cecum to the anus, and some of the intestinal tissue was cut into approximately $4 \mathrm{~mm}$ lengths. The tissue was embedded in paraffin and fixed with $4 \%$ buffered paraformaldehyde for 12 to $24 \mathrm{~h}$.

\section{Survival rate analysis}

The rats were also divided into four groups: control group, sepsis group, sepsis+sh-IL-9 group, and sepsis+ IL-9 group ( $n=10$ in each group). After sepsis induction by CLP, rats in these groups were observed and recorded daily for 14 days to assess the survival rate.

\section{Cytokine and flow cytometry measurement}

The human serum concentrations of IL-9 and D-lactate were measured by human enzyme-linked immunosorbent assay (ELISA) kits. The IL-9-producing CD4(+) T cells proportions of serum or intestinal tissue were measured by flow cytometry [FITC-CD4 antibody (eBioscience, 11-0400-82), IL-9 antibody (eBioscience, PA5-47584), Donkey anti-sheep antibody alexa fluor647 (abcam, ab150179)]. The serum was collected from the rats and the concentrations of IL-9 (Cusabio, Wuhan,
China) and D-lactate (MyBioSource, San Diego, CA, USA) were also measured by ELISA kits according to the manufacturer's instructions.

\section{Western blot}

For immunoblotting, the intestinal tissue was lysed in radio immunoprecipitation assay lysis buffer. The lysate was separated in sodium dodecyl sulfate-polyacrylamide gel electrophoresis and transferred to a nitrocellulose membrane. The protein concentration of lysate was detected using a bicinchoninic acid kit (Bio-Rad Laboratories, Hercules, CA, USA). The blot of lysates was incubated with the following antibodies: IL-9 antibody (Abcam, diluted in 1:1000), D-lactate antibody (abcam, diluted in 1:1000), zonula occludens $1(\mathrm{ZO}-1)$ antibody (proteintech, diluted in 1:1000), horseradish peroxidase (HRP)-labeled anti-rabbit antibody (Beyotime, Shanghai, China), GAPDH antibody (ZEN-bio, diluted in 1:5000), and HRP-labeled anti-mouse antibody (Beyotime, Shanghai, China). Images were acquired with Tanon enhanced chemiluminescence substrate (Tanon, Shanghai, China), using Tanon 5200 imaging system (Tanon, Shanghai, China).

\section{Histopathology}

Following rat sacrifice, the intestinal tissue was collected and fixed in $4 \%$ formalin and embedded in paraffin. Sections of tissue $(4 \mu \mathrm{m})$ were stained with hematoxylin and eosin (HE) and observed by Nikon H550S microscope and Nikon DS-Ri2 imaging system (Nikon, Japan). The Chiu score was used to assess the intestinal injury.

\section{Apoptosis}

Apoptosis of intestinal epithelial cells was determined by terminal deoxynucleotidyltransferase-mediated deoxyuridine triphosphate nick-end labeling (TUNEL) assay. Intestine tissue samples were fixed in $4 \%$ buffered paraformaldehyde, embedded and cut into $4 \mu \mathrm{m}$ sections. Then the sections were stained with TUNEL kit (KeyGenBiotech, Nanjing, China) according to the manufacturer's instructions. Each section was observed under a light microscope and the cells in four randomly selected regions were counted. The positive apoptotic nuclei were brown in color.

\section{Intestinal permeability}

Intestinal mucosal permeability was measured by fluorescence tracing method. A section of intestine about 15 $\mathrm{cm}$ long was chosen and ligated at one end, and then the tracer (EZ-Link NHS-Biotin) (Thermo Fisher Scientific, MA, USA) was diluted to $2 \mathrm{mg} / \mathrm{ml}$ and injected into the distal intestinal lumen slowly. A segment of intestine about $5 \mathrm{~cm}$ long was taken and fixed in $4 \%$ paraformaldehyde solution for $3 \mathrm{~h}$, then washed with phosphate 
buffer solution 3 times and sliced. The tissue was incubated with streptavidin at room temperature for $30 \mathrm{~min}$. The distribution of the fluorescent tracer in intestinal tissue was observed by a laser confocal microscope.

\section{Statistical analysis}

The Kolmogorov-Smirnov test was first performed to test the normal distribution of the data. Normally distributed data were expressed as the means \pm standard deviation and were compared by $t$ tests. Non-normally distributed data were expressed as the medians (interquartile ranges) and were compared by the MannWhitney $U$ test or the Kruskal-Wallis test. Categorical variables were presented as absolute numbers or percentages and were analyzed using the $\chi^{2}$ test or Fisher's exact test. To take into account the repeated nature of the variables, analysis of variance (ANOVA) and LSD post hoc tests for repeated measurements of the general linear model were implemented. Scatterplots and a correlation analysis were performed to evaluate the relevance between IL-9-producing CD4(+) T cells, IL-9, and D-lactate. Receiver operating characteristic (ROC) curves were used to evaluate the associations between IL-9producing CD4(+) T cells, IL-9, and 28-day mortality in patients. Survival curves for up to 14 days after sepsis induction of rats were generated using the Kaplan-Meier method and were compared by the log-rank test. IBM Statistical Package for the Social Sciences (SPSS, version 22.0, NY, USA) software was used for statistical analysis, and two-sided $P<0.05$ was considered statistically significant. The statistical methods of this study were reviewed by Qiao Liu, a biostatistician from the Center for Disease Control and Prevention of Jiangsu Province in China.

\section{Results}

A total of 36 septic patients and 15 non-septic controls were enrolled in this study. The demographic data and clinical parameters of the patients on admission are presented in Table 1 . The 28-day mortality was $27.8 \%$ (10/ $36)$ in these septic patients. Thirteen patients $(13 / 36$, $36.1 \%)$ received continuous renal replacement therapy, and 33 patients $(33 / 36,91.7 \%)$ received mechanical ventilation during hospital stay.

The serum of the septic patients had IL-9-producing CD4(+) T cell percentages, IL-9, and D-lactate levels that were significantly higher than those of the nonseptic controls during 7 days of ICU admission (Fig. 1a, $P<0.01)$. The results of scatterplots and correlation analyses were shown in Fig. 1b. IL-9 levels were positively correlated with IL-9-producing CD4(+) T cell percentages on day $1\left(R^{2}=0.416, P<0.001\right)$, day $3\left(R^{2}=0.351, P\right.$ $<0.001)$, and day $7\left(R^{2}=0.305, P<0.001\right)$ of admission. IL-9 levels were also positively correlated with D-lactate levels on day $1\left(R^{2}=0.486, P<0.001\right)$, day $3\left(R^{2}=0.443\right.$, $P<0.001)$, and day $7\left(R^{2}=0.337, P<0.001\right)$. Moreover, IL-9-producing CD4(+) $\mathrm{T}$ cell percentages were only positively correlated with D-lactate levels on day $1\left(\mathrm{R}^{2}=\right.$ $0.366, P<0.001)$. In addition, non-surviving septic patients had significantly higher serum IL-9-producing CD4(+) T cell percentages, IL-9, and D-lactate levels compared with survivors (Fig. 1 c, $P<0.01$ ). ROC curve results demonstrated that both IL-9-producing CD4(+) $\mathrm{T}$ cell percentages and IL-9 levels on day 1 of admission

Table 1 Demographic data and clinical parameters on admission

\begin{tabular}{|c|c|c|c|c|c|}
\hline & $\begin{array}{l}\text { Sepsis } \\
(n=36)\end{array}$ & & $\begin{array}{l}\text { Control } \\
(n=15)\end{array}$ & & $P$ value \\
\hline Age (years) & $67.00(63.25-72.75)$ & & $65.20(59.00-69.25)$ & & 0.267 \\
\hline Sex (male:female) & $20: 16$ & & $9: 6$ & & 0.770 \\
\hline \multirow[t]{7}{*}{ Disease categories $(n, \%)$} & Etiology of sepsis & & Etiology of ICU admission & & \\
\hline & Abdominal infection & 19 (52.8\%) & Multiple trauma & $4(26.7 \%)$ & / \\
\hline & Thoracic/pulmonary infection & $6(16.7 \%)$ & Acute cardiac dysfunction & $3(20.0 \%)$ & / \\
\hline & Blood stream infection & $4(11.1 \%)$ & Cerebral hemorrhage & $3(20.0 \%)$ & / \\
\hline & Urinary infection & $3(8.3 \%)$ & Acute kidney injury & $2(13.3 \%)$ & \\
\hline & Mucocutaneous infection & $2(5.6 \%)$ & ARDS & $2(13.3 \%)$ & / \\
\hline & Other & $2(5.6 \%)$ & Hemorrhagic shock & $1(6.7 \%)$ & / \\
\hline BMI $\left(\mathrm{kg} / \mathrm{m}^{2}\right)$ & $24.22(21.05-26.02)$ & & $24.15(20.83-25.80)$ & & 0.852 \\
\hline APACHE ॥ & $23.00(19.00-27.50)$ & & $24.50(18.50-29.00)$ & & 0.632 \\
\hline SOFA & $9.50(8.00-11.75)$ & & $11.00(9.50-13.00)$ & & 0.113 \\
\hline ICU days & $9.00(5.25-13.00)$ & & $8.50(5.50-12.75)$ & & 0.323 \\
\hline Death $(n, \%)$ & $10(27.8 \%)$ & & $3(20.0 \%)$ & & 0.730 \\
\hline
\end{tabular}

ICU intensive care unit, ARDS acute respiratory distress syndrome, BMI body mass index, APACHE II Acute Physiology and Chronic Health Evaluation II, SOFA Sequential Organ Failure Assessment 


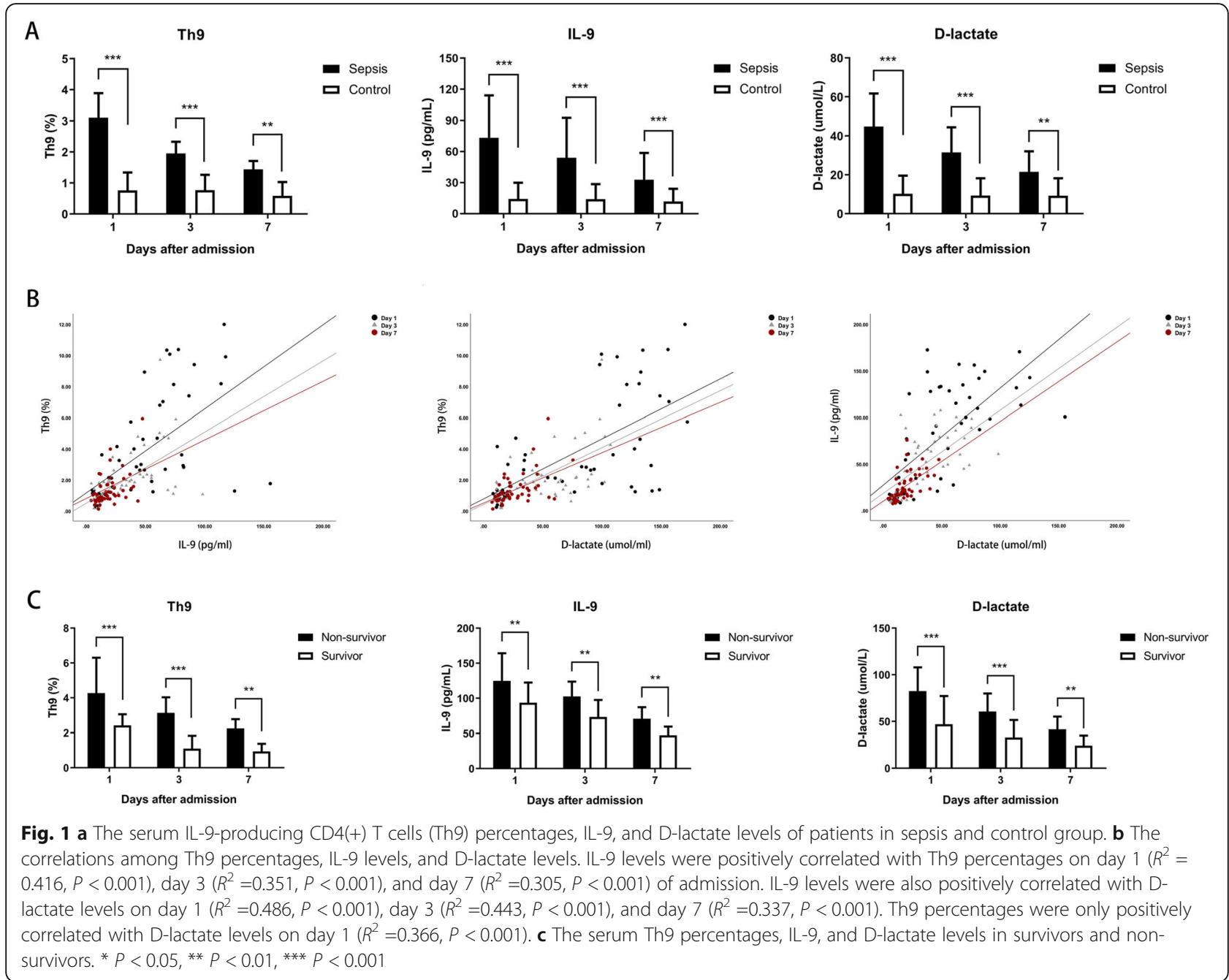

had a high predictive value of 28-day mortality in septic patients (Fig. 2a). The area under the curves (AUCs) of IL-9-producing CD4(+) $\mathrm{T}$ cell percentages and IL-9 levels were $0.887(P<0.001)$ and $0.835(P=0.002)$, respectively. The IL-9-producing CD4(+) T cell percentages (AUC $=0.694, P=0.312$ ) and IL-9 levels (AUC = $0.750, P=0.194$ ) of non-septic patients had no significant predictive value of 28 -day mortality (Fig. $2 \mathrm{~b}$ ). The results suggested that IL-9-producing CD4(+) T cells and IL-9 are associated with prognosis and damage to the intestinal mucosal barrier in septic patients.

To functionally confirm the role of IL-9-producing CD4(+) T cells and IL-9 in the intestinal pathology, we used a rat sepsis model. A total of 48 SD rats were randomly assigned to four groups as follows: control group, sepsis group, sepsis+sh-IL-9 group, and sepsis+ IL-9 group. As shown in Fig. 3a, the serum percentages of IL-9-producing CD4(+) T cells of the control group were significantly lower than that of the sepsis group $(0.43 \% \pm 0.03 \%$ vs. $1.58 \% \pm 0.41 \%, P=0.021)$ or the
sepsis+IL-9 group $(0.43 \% \pm 0.03 \%$ vs. $3.53 \% \pm 0.90 \%, P<$ $0.001)$. The serum percentages of IL-9-producing CD4(+) T cells of the sepsis+IL-9 group were significantly higher than that of the sepsis group $(3.53 \% \pm$ $0.90 \%$ vs. $1.58 \% \pm 0.41 \%, P=0.001)$ or the sepsis+sh-IL-9 group $(3.53 \% \pm 0.90 \%$ vs. $0.80 \% \pm 0.02 \%, P<0.001)$. The serum IL-9 and D-lactate levels in the control group were significantly lower than that of the sepsis group and sepsis+IL-9 group (Fig. 3b, $P<0.01$ ). Moreover, the serum levels of the two markers were gradually increased in the sepsis+sh-IL-9 group, sepsis group, and sepsis+IL-9 group (Fig. 3b, $P<0.01$ ). Survival rate analysis also showed that the survival rate of rats was gradually decreased in the control group (100\%), the sepsis+ sh-IL-9 group (70\%), the sepsis group (50\%), and the sepsis+IL-9 group (20\%) (Fig. 3c, $P<0.001$ ).

The differences of IL-9-producing CD4(+) T cell percentages, IL-9, and D-lactate levels in intestinal tissue of rats among the four groups were shown in Fig. 4. As shown in Fig. 4a, the percentages of tissue IL-9- 

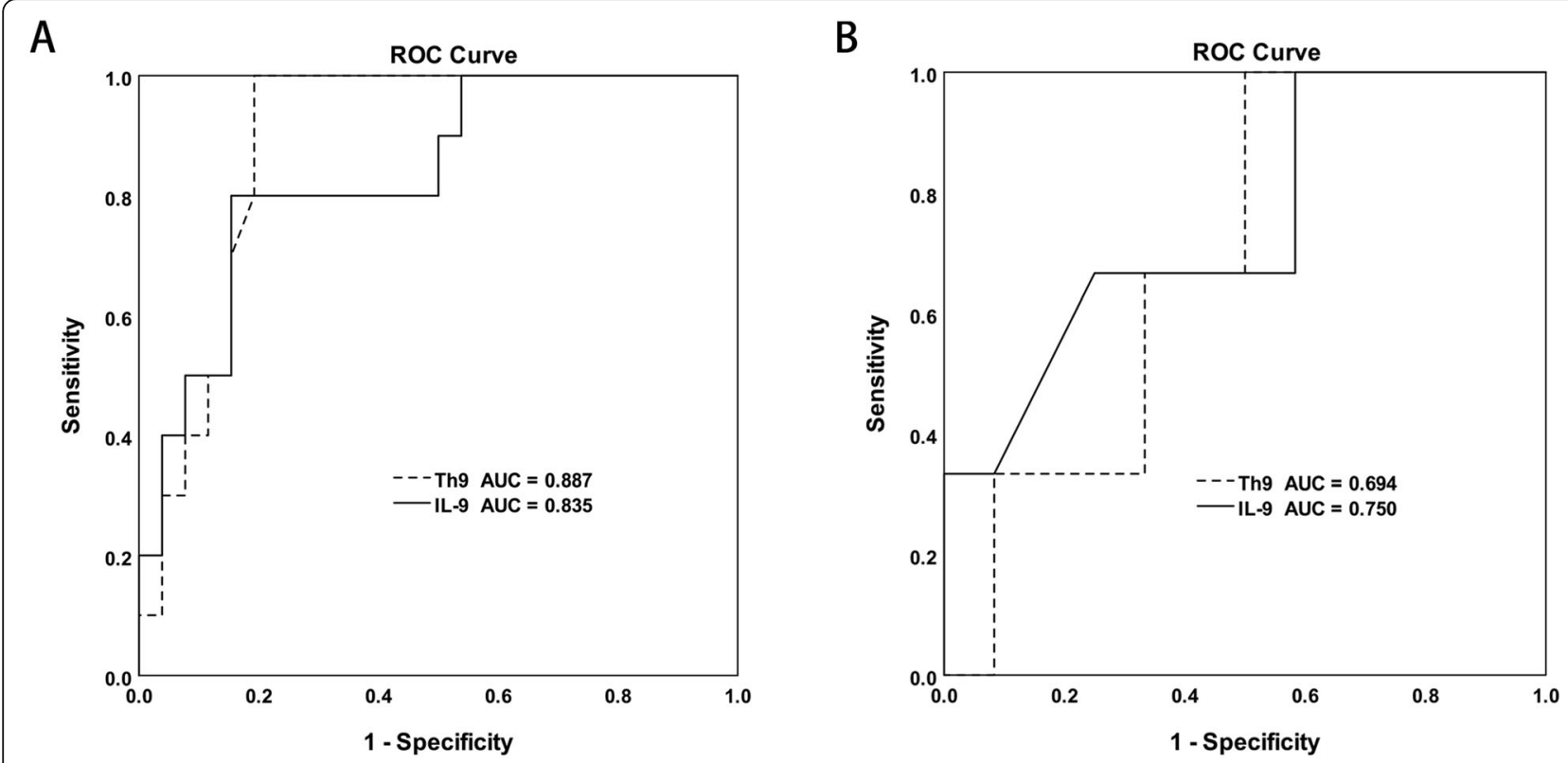

Fig. 2 Receiver operating characteristic (ROC) curves were used to evaluate the value of IL-9-producing CD4(+) T cells (Th9) percentages and IL-9 levels on day 1 of admission in predicting 28-day mortality in septic (a) and non-septic (b) patients. A. The area under the curves (AUCs) of Th9 percentages and IL-9 levels were $0.887(P<0.001)$ and $0.835(P=0.002)$, respectively. $\mathbf{b}$ The AUCs of Th9 percentages and IL-9 levels were $0.694(P$ $=0.312)$ and $0.750(P=0.194)$, respectively

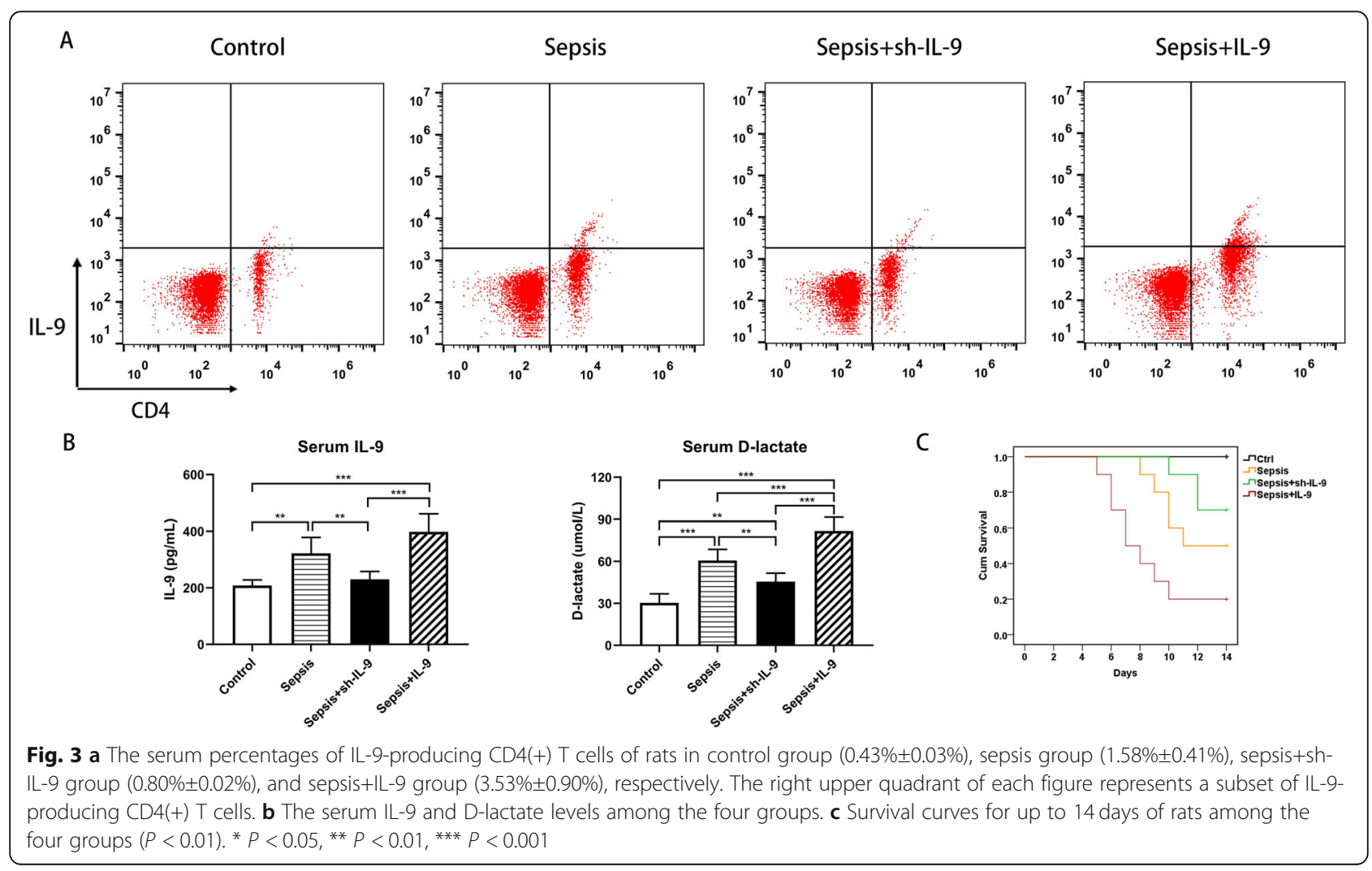


producing CD4(+) T cells in the control group were significantly lower than that in the sepsis group $(0.22 \% \pm$ $0.04 \%$ vs. $1.02 \% \pm 0.07 \%, P<0.001)$ or the sepsis+IL-9 group $(0.22 \% \pm 0.04 \%$ vs. $1.57 \% \pm 0.10 \%, P<0.001)$. The percentages of tissue IL-9-producing CD4(+) T cells in the sepsis group were significantly higher than that in the sepsis+sh-IL-9 group $(1.02 \% \pm 0.07 \%$ vs. $0.16 \% \pm 0.01 \%$, $P<0.001)$, whereas IL-9-producing CD4(+) T cell percentages significantly lower than that in the sepsis+IL-9 group $(1.02 \% \pm 0.07 \%$ vs. $1.57 \% \pm 0.10 \%, P<0.01)$. Western blot analysis (Fig. 4b, c) showed that the expression levels of IL-9 and D-lactate in intestinal tissue of controls were significantly lower than that of the sepsis group $(P<0.05)$ and the sepsis+IL-9 group $(P<0.001)$. Compared with the sepsis group, the expression levels of IL-9 and D-lactate were significantly increased in the sepsis+IL-9 group $(P<0.01)$, whereas those were significantly decreased in the sepsis+sh-IL-9 group $(P<0.001)$. The ZO-1 levels of intestinal tissue in controls were significantly higher than that of the other three groups (Fig. $4 b, c, P<0.001)$. Compared with the sepsis group, the ZO-1 levels were significantly increased in the sepsis+ sh-IL-9 group $(P<0.01)$, whereas those were significantly decreased in the sepsis+IL-9 group $(P<0.001)$.

HE staining (Fig. 5a) showed that intestinal mucosa was normal, and the epithelium was intact in non-septic controls. In the sepsis group, lamina propria hemorrhage and inflammatory cell infiltration were also observed. The sepsis-induced intestinal mucosal injuries were significantly improved or aggravated by IL- 9 interference or IL-9 overexpression, respectively (Fig. 5a). Morphological injuries were assessed by Chiu's score (Fig. 5b, $P$ $<0.05)$. TUNEL apoptosis detection also demonstrated similar findings (Fig. 5c). The positive apoptotic nuclei were brown in color. The percentage of apoptotic cells in intestinal mucosa was gradually increased in the control group, sepsis+sh-IL-9 group, sepsis group, and sepsis+IL-9 group (Fig. 5d, $P<0.01$ ).

Intestinal mucosal permeability was detected by a laser confocal microscope (Fig. 6). The distribution of fluorescent tracer (green color) was observed only on the surface of intestinal mucosa in non-septic controls (Fig. 6a). In the sepsis group, the tracer penetrated into the muscular layer of the intestinal mucosa (Fig. 6a). After IL-9 interference, the tracer only penetrated into the epithelial layer of intestinal mucosa, while after IL-9 overexpression, the tracer penetrated deeply into the intestinal submucosa (Fig. 6a). Comparisons of integrated optical density (IOD) values were shown in Fig. 6b. The IOD values of fluorescent tracer were gradually increased in the control group, sepsis+sh-IL-9 group, sepsis group, and sepsis+IL-9 group $(P<0.01)$. Moreover, we also compared the differences of tracer penetration depth among

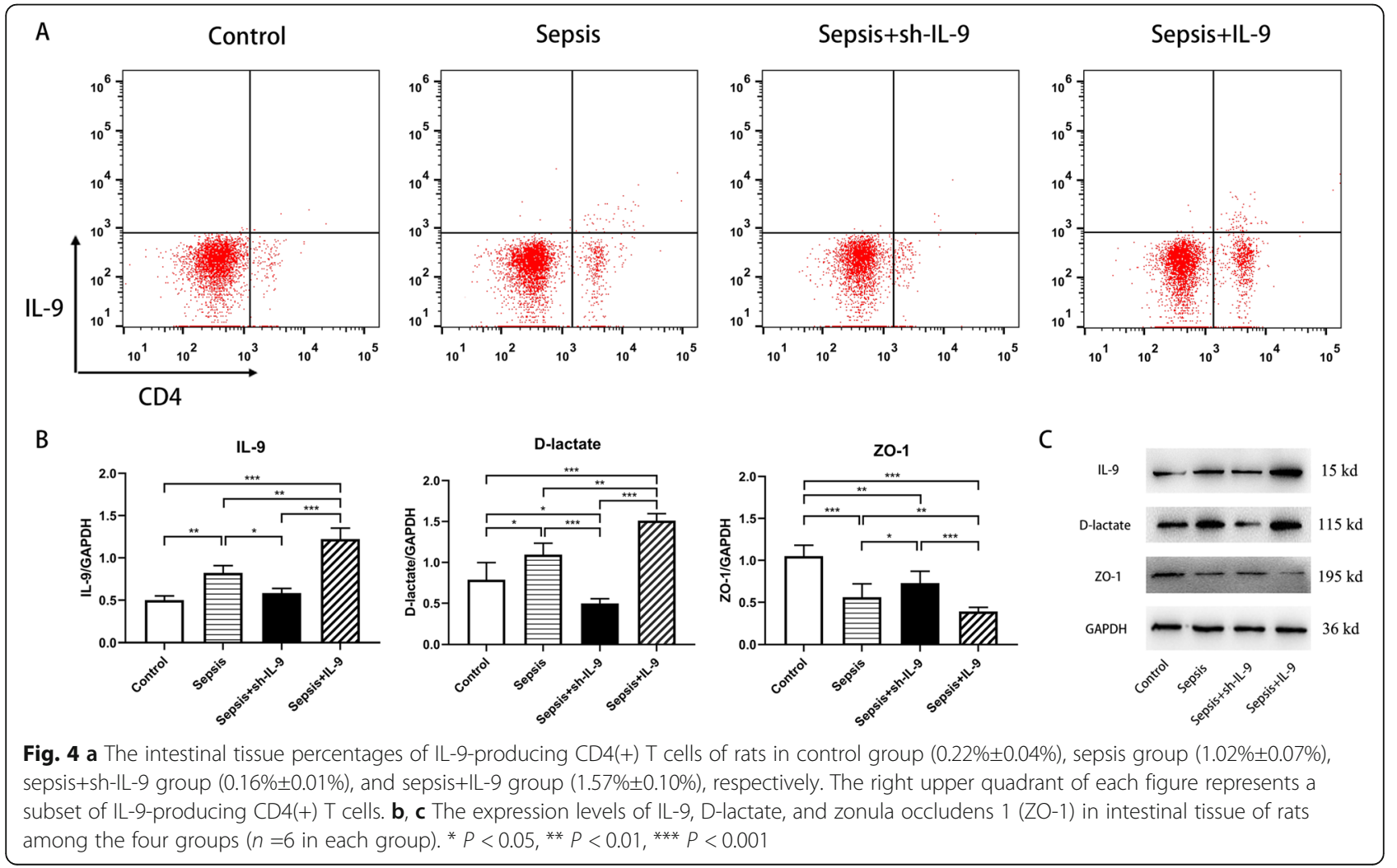




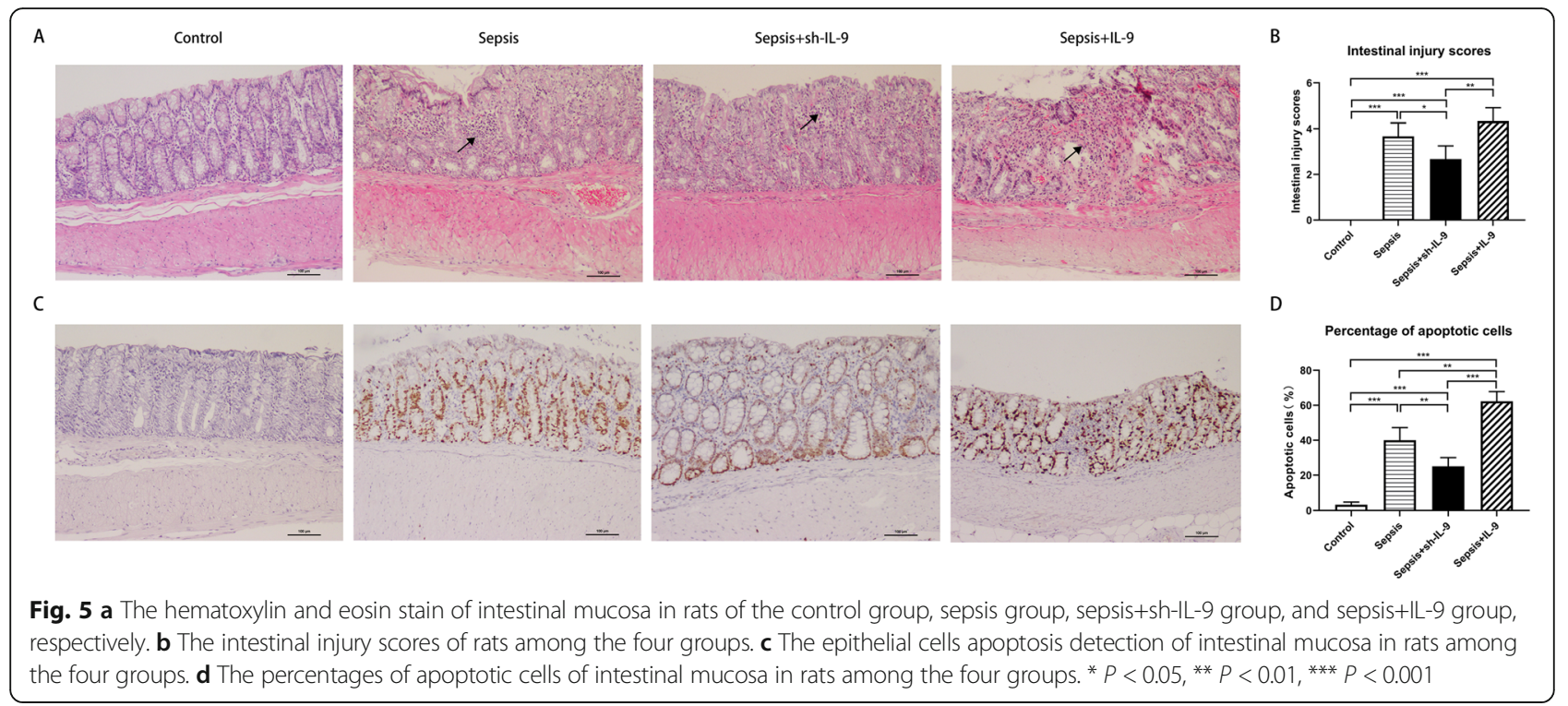

these groups. The penetration depth of tracer was gradually increased in the control group, sepsis+sh-IL- 9 group, sepsis group, and sepsis+IL-9 group (Fig. 6c, $P<0.05$ ).

\section{Discussion}

It is known that IL-9-producing CD4(+) T cells and IL-9 are associated with inflammatory bowel disease, tumor, and autoimmune diseases [7, 9, 10]. However, the roles of IL-9-producing CD4(+) T cells and IL-9 in sepsis are not clear. Intestinal mucosal barrier injury is one of the important potentiators of sepsis $[5,6]$. This study firstly proved that IL-9-producing CD4(+) T cells and IL-9 were also closely related to the barrier injury and mortality in sepsis. We found that the serum IL-9-producing CD4(+) T cell percentages, IL-9, and D-lactate levels were significantly higher in septic patients or rats than that in controls. IL-9-producing CD4(+) T cell percentages and IL-9 levels were positively correlated with Dlactate levels in septic patients. IL-9-producing CD4(+) $\mathrm{T}$ cell percentages and IL-9 levels also had a high predictive value of 28 -day mortality. The non-survivors had significantly higher serum IL-9-producing CD4(+) T cell

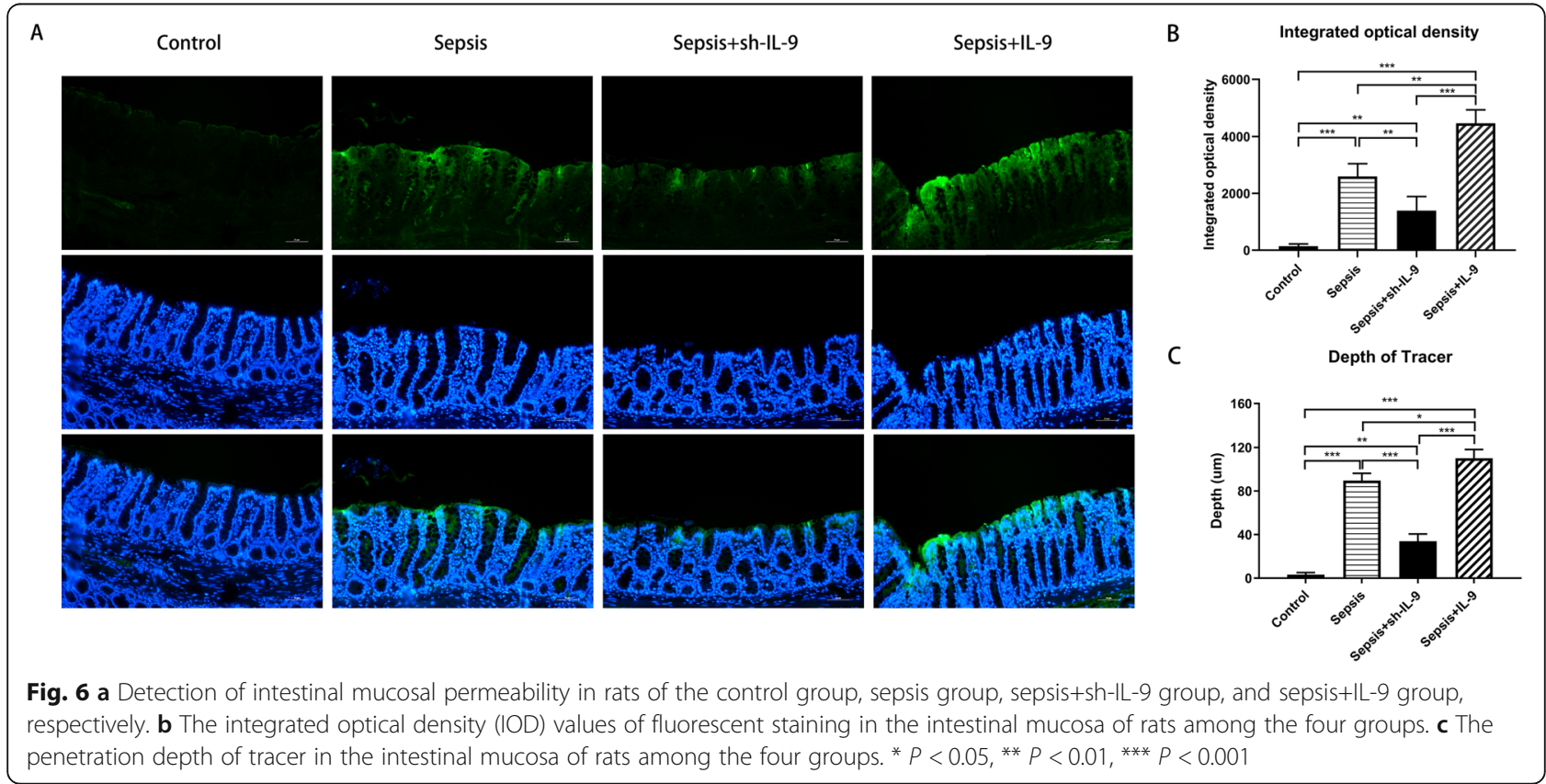


percentages, IL-9, and D-lactate levels compared with survivors. In septic rats, IL-9 increased the expression levels of D-lactate, whereas that decreased the expression levels of ZO-1. Moreover, the barrier injury was aggravated or alleviated by increasing or interfering with IL-9 expression, respectively. Survival rate analysis also showed that IL-9 decreased the 14-day survival rate of septic rats.

The intestinal tract is considered a vital immune organ. Acute intestinal barrier damage and systemic infection are a vicious cycle in critical diseases, especially in sepsis $[1,19,20]$. Our previous studies showed that $\mathrm{T}$ helper lymphocytes were associated with gastrointestinal injury and immune dysregulation in sepsis $[20,21]$. In addition to the conventional Th1/Th2 and Th17/Treg lymphocytes, Th9 cells also contribute to chronic intestinal and airway inflammation [7-10, 12, 14]. Renga et al. [8] reported that IL-9 and mucosal mast cells contributed to barrier function loss, dissemination, and inflammatory dysbiosis in experimental leaky gut models and human celiac disease. Feng et al. [9] found that serum IL-9 level was upregulated significantly and correlated with disease severity in patients with Crohn's Disease. Wang [14] and Zhao's [22] research showed that tumor necrosis factor-like ligand 1A could destroy the mucosal barrier by promoting Th9 and IL-9 overexpression in mice with chronic colitis. Consequently, it is reasonable to speculate that Th9 and IL-9 may be related to the barrier injury in sepsis. Our findings suggest that IL9 overexpression can increase inflammatory cell infiltration, epithelial cell apoptosis, and mucosal permeability of the intestine in septic rats. D-lactate and ZO-1 are common biomarkers of mucosal barrier function [17, 22]. The D-lactate level was positively correlated with the degree of intestinal barrier injury, whereas the ZO-1 was opposite $[5,6]$. In both animal models and humans of our study, IL-9-producing CD4(+) T cell percentages and IL-9 levels were positively correlated with the concentrations of D-lactate. In animal models, IL-9 increased the expression level of D-lactate, whereas that decreased the expression level of ZO-1. These results suggest that IL-9-producing CD4(+) T cells and IL-9 could be potential therapeutic targets of intestinal mucosal injury in sepsis. Our findings also support this conclusion, because the mucosal injury was improved after IL-9 interference in septic rats.

Intestinal mucosal barrier damage and enterogenous infection contribute to the high mortality in sepsis $[1,3]$. In other words, non-surviving septic patients tend to have more severe barrier damage compared with survivors. In our study, the non-survivors had significantly higher serum IL-9-producing CD4(+) T cell percentages, IL-9, and D-lactate levels compared with survivors. ROC curve results demonstrated that both IL-9-producing
CD4(+) T cell percentages and IL-9 levels had a high predictive value of 28-day mortality. Survival rate analysis of animal models also showed that IL-9 decreased the 14-day survival rate of sepsis rats. Therefore, inhibition of Th9 cells differentiation or IL-9 expression may be a novel strategy to improve survival in sepsis. We are now conducting a clinical study of this project (not yet published). Th9 cells are derived from naive $\mathrm{T}$ cells in the presence of transforming growth factor $\beta$ and interleukin-4 $[7,10]$. In addition to IL-9, Th9 cells also express IL-10, IL-21, and other cytokines [10]. Interestingly, we found that Th9 percentages of serum and intestinal tissue were decreased after IL-9 interference, whereas IL-9-producing CD4(+) $\mathrm{T}$ cell percentages increased after IL-9 overexpression in septic rats. The phenomenon revealed that IL-9-producing $\mathrm{CD} 4(+) \mathrm{T}$ cells and IL-9 might have a feedback relationship through some potential pathways, but the mechanism was not clear and remained to be tested by further research.

Although numerous studies have elucidated the role of IL-9 in pro-inflammatory activity, some studies found that IL-9 also had anti-inflammatory effects. IL-9 could protect mice from Gram-negative bacterial shock by suppressing TNF-alpha, IL-12, and IFN-gamma, or inhibit oxidative burst and TNF-alph release in lipopolysaccharide-stimulated human monocytes $[13,23$, 24]. In the present study, we found that IL-9 mainly played a pro-inflammatory role in the intestinal mucosal injury of sepsis. IL-9 was associated with epithelial cell apoptosis and increased mucosal permeability in septic rats. However, whether IL-9-producing CD4(+) T cells and IL-9 have protective effects in sepsis need confirmation by more studies.

Some limitations of this study should be discussed. Due to our single-center design and small sample size, the results may not be generalizable, and the accuracy should be confirmed by large-scale clinical prospective studies. Moreover, Th9 cells can secrete a variety of cytokines, and IL-9 is also secreted by other cells. The roles of these incidental cytokines or cells were not investigated in our study. In addition, only the IL-9 pathway instead of the IL-9-producing CD4(+) T cells was intervened in this study. The specific mechanism by which IL-9-producing CD4(+) T cells are involved in the mucosal barrier injury of sepsis needs further research.

\section{Conclusions}

This study suggested that IL-9 is closely related to the intestinal mucosal barrier injury in sepsis. Patients with sepsis had increased IL-9 levels compared to patients without sepsis. IL-9-producing CD4(+) T cells and IL-9 levels were positively correlated with D-lactate levels and had a high predictive value of 28-day mortality in septic 
patients. The intestinal barrier injury was aggravated or alleviated by increasing or interfering with the expression of IL-9, respectively. IL-9 also increased the mortality of sepsis in animal models. Further studies are warranted to investigate whether IL-9 blockade has the potential to improve the mucosal barrier injury in sepsis.

\section{Abbreviations}

Th: T helper; Treg: T regulatory; Th9: IL-9-producing CD4(+) T helper; IL9: Interleukin-9; ICU: Intensive care unit; APACHE II: Acute Physiology and Chronic Health Evaluation II; SOFA: Sequential Organ Failure Assessment; CLP: Cecum ligation and puncture; AAV: Adeno-associated virus;

ELISA: Enzyme-linked immunosorbent assay; ZO-1: Zonula occludens 1; HRP: Horseradish peroxidase; HE: Hematoxylin and eosin; TUNEL: Terminal deoxynucleotidyltransferase-mediated deoxyuridine triphosphate nick-end labeling; ANOVA: analysis of variance; ROC: Receiver operating characteristic; SPSS: Statistical Package for the Social Sciences

\section{Acknowledgements}

The authors thank Fan Qiu, Yuan Fang, Qian-Kun Shi, Wen-Xiu Chen, and Wen-Hao Zhang of Nanjing first hospital for their contributions to this study. In addition, Jia-Kui Sun and his family especially thank Sun XP for her meticulous care and support during the past 10 years.

\section{Authors' contributions}

Sun JK and Zhou J contributed equally. Sun JK, Zhou J, Zhou SM, and Mu XW designed the study and drafted the manuscript. Sun JK and Wang $X$ collected the clinical samples and data. Sun JK, Zhou J, and Shen X performed the experiments and statistical analysis. Sun XP and Zhu DM participated in its design and coordination and helped to perform the experiments. The authors read and approved the final manuscript.

\section{Funding}

This study was supported by the National Natural Science Foundation of China (No. 81701881) and the Nanjing Medical Science and Technology Development Foundation (No. YKK17102).

\section{Availability of data and materials}

Not applicable.

\section{Declarations}

\section{Ethics approval and consent to participate}

The study protocol was approved by the Institutional Ethics Committee of Nanjing First Hospital (Approval Number: KY20180713-01), and informed consent was obtained from each patient's first-degree relatives according to the Declaration of Helsinki. This study was also registered at Clinical Trials.gov (ID: NCT03791866)

\section{Consent for publication}

Yes from all authors.

\section{Competing interests}

The authors declare that they have no competing interests.

\section{Author details}

'Department of Geriatrics Intensive Care Unit, The First Affiliated Hospital of Nanjing Medical University (Jiangsu Province People's Hospital), 300 Guangzhou Road, Nanjing 210029, Jiangsu Province, China. 'Department of Critical Care Medicine, Nanjing First Hospital, Nanjing Medical University, 68 Changle Road, Nanjing 210006, Jiangsu Province, China. ${ }^{3}$ Department of General Office, Productivity Center of Jiangsu Province, 175 Longpan Road, Nanjing 210042, Jiangsu Province, China.

Received: 7 March 2021 Accepted: 25 April 2021

Published online: 03 May 2021

\section{References}

1. Rhodes A, Evans LE, Alhazzani W, Levy MM, Antonelli M, Ferrer R, et al. Surviving sepsis campaign: international guidelines for management of sepsis and septic shock: 2016. Crit Care Med. 2017:45(3):486-552. https://doi. org/10.1097/CCM.0000000000002255.

2. Singer M, Deutschman CS, Seymour CW, Shankar-Hari M, Annane D, Bauer M, et al. The Third International Consensus Definitions for Sepsis and Septic Shock (Sepsis-3). JAMA. 2016;315(8):801-10. https://doi.org/10.1001/jama.201 6.0287 .

3. Perner A, Rhodes A, Venkatesh B, Angus DC, Martin-loeches I, Preiser JC, et al. Sepsis: frontiers in supportive care, organisation and research. Intensive Care Med. 2017;43(4):496-508. https://doi.org/10.1007/s00134-01 7-4677-4.

4. Li J, Li M, Su L, Wang H, Xiao K, Deng J, et al. Alterations of T helper lymphocyte subpopulations in sepsis, severe sepsis, and septic shock: a prospective observational study. Inflammation. 2015;38(3):995-1002. https:// doi.org/10.1007/s10753-014-0063-3.

5. Fay $\mathrm{KT}$, Ford $\mathrm{ML}$, Coopersmith $\mathrm{CM}$. The intestinal microenvironment in sepsis. Biochim Biophys Acta Mol Basis Dis. 2017;1863(10 Pt B):2574-83.

6. Gupta DL, Bhoi S, Mohan T, Galwnkar S, Rao DN. Coexistence of Th1/Th2 and Th17/Treg imbalances in patients with post traumatic sepsis. Cytokine. 2016;88:214-21. https://doi.org/10.1016/j.cyto.2016.09.010.

7. Nalleweg N, Chiriac MT, Podstawa E, Lehmann C, Rau T, Atreya R, et al. IL-9 and its receptor are predominantly involved in the pathogenesis of UC. Gut. 2015;64(5):743-55. https://doi.org/10.1136/gutjnl-2013-305947.

8. Renga G, Moretti S, Oikonomou V, Borghi M, Zelante T, Paolicelli G, et al. IL9 and Mast Cells Are Key Players of Candida albicans Commensalism and Pathogenesis in the Gut. Cell Rep. 2018;23(6):1767-78. https://doi.org/10.101 6/j.celrep.2018.04.034.

9. Feng T, Chen B, Li L, Huang S, Ben-Horin S, Qiu Y, et al. Serum interleukin 9 levels predict disease severity and the clinical efficacy of infliximab in patients with Crohn's disease. Inflamm Bowel Dis. 2017;23(10):1817-24. https://doi.org/10.1097/MIB.0000000000001172.

10. Schmitt E, Klein M, Bopp T. Th9 cells, new players in adaptive immunity. Trends Immunol. 2014;35(2):61-8. https://doi.org/10.1016/j.it.2013.10.004

11. Zundler S, Schillinger D, Fischer A, Atreya R, López-Posadas R, Watson A, et al. Blockade of aE $\beta 7$ integrin suppresses accumulation of CD8+and Th9 lymphocytes from patients with IBD in the inflamed gut in vivo. Gut. 2017; 66(11):1936-48. https://doi.org/10.1136/qutjnl-2016-312439.

12. Ciccia F, Guggino G, Ferrante A, et al. Interleukin-9 overexpression and Th9 polarization characterize the inflamed gut, the synovial tissue, and the peripheral blood of patients with psoriatic arthritis. Arthritis Rheumatol. 2016;68(8):1922-31.

13. Yuan A, Yang H, Qi H, Cui J, Hua W, Li C, et al. IL-9 antibody injection suppresses the inflammation in colitis mice. Biochem Biophys Res Commun. 2015;468(4):921-6. https://doi.org/10.1016/j.bbrc.2015.11.057.

14. Wang D, Li H, Duan YY, Han F, Luo YX, Wu MY, et al. TL1A modulates the severity of colitis by promoting Th9 differentiation and IL-9 secretion. Life Sci. 2019;231:116536. https://doi.org/10.1016/j.lfs.2019.06.011.

15. Rampal R, Awasthi A, Ahuja V. Retinoic acid-primed human dendritic cells inhibit Th9 cells and induce Th1/Th17 cell differentiation. J Leukoc Biol. 2016;100(1):111-20. https://doi.org/10.1189/jlb.3VMA1015-476R.

16. Lu J, Zhang L, Cheng L, He S, Zhang Y, Yan J, et al. Xijiao Dihuang decoction improves prognosis of sepsis via inhibition of aerobic glycolysis. Biomed Pharmacother. 2020;129:110501. https://doi.org/10.1016/j.biopha.202 0.110501 .

17. Tu H, Lai X, Li J, Huang L, Liu Y, Cao J. Interleukin-26 is overexpressed in human sepsis and contributes to inflammation, organ injury, and mortality in murine sepsis. Crit Care. 2019;23(1):290. https://doi.org/10.1186/s13054-01 9-2574-7.

18. Tudurí E, Glavas MM, Asadi A, Baker RK, Ellis CE, Soukhatcheva G, et al. AAV GCG-EGFP, a new tool to identify glucagon-secreting a-cells. Sci Rep. 2019; 9(1):10829. https://doi.org/10.1038/s41598-019-46735-2.

19. Sun JK, Liu Y, Zou L, Zhang WH, Li JJ, Wang Y, et al. Acute gastrointestinal injury in critically ill patients with COVID-19 in Wuhan, China. World J Gastroenterol. 2020;26(39):6087-97. https://doi.org/10.3748/wjg.v26.i39.6087.

20. Sun JK, Zhang WH, Chen WX, Wang X, Mu XW. Effects of early enteral nutrition on Th17/Treg cells and IL-23/IL-17 in septic patients. World J Gastroenterol. 2019;25(22):2799-808. https://doi.org/10.3748/wjg.v25.i22.2 799.

21. Sun JK, Yuan ST, Mu XW, Zhang WH, Liu Y, Zou L, et al. Effects of early enteral nutrition on $T$ helper lymphocytes of surgical septic patients: a retrospective observational study. Medicine (Baltimore). 2017:96(32):e7702. https://doi.org/10.1097/MD.0000000000007702. 
22. Zhao C, Wang D, Wu M, Luo Y, Yang M, Guo J, et al. Tumor necrosis factor ligand-related molecule $1 \mathrm{~A}$ affects the intestinal mucosal barrier function by promoting Th9/interleukin-9 expression. J Int Med Res. 2020;48(6): 300060520926011. https://doi.org/10.1177/0300060520926011.

23. Li H, Nourbakhsh B, Cullimore M, Zhang GX, Rostami A. IL-9 is important for T-cell activation and differentiation in autoimmune inflammation of the central nervous system. Eur J Immunol. 2011;41(8):2197-206. https://doi. org/10.1002/eji.201041125.

24. Goswami R, Kaplan MH. A brief history of IL-9. J Immunol. 2011;186(6):32838. https://doi.org/10.4049/jimmunol.1003049.

\section{Publisher's Note}

Springer Nature remains neutral with regard to jurisdictional claims in published maps and institutional affiliations.

Ready to submit your research? Choose BMC and benefit from:

- fast, convenient online submission

- thorough peer review by experienced researchers in your field

- rapid publication on acceptance

- support for research data, including large and complex data types

- gold Open Access which fosters wider collaboration and increased citations

- maximum visibility for your research: over $100 \mathrm{M}$ website views per year

At BMC, research is always in progress.

Learn more biomedcentral.com/submissions 